\title{
OSCILLATORY AND ASYMPTOTIC BEHAVIOUR OF SECOND ORDER NONLINEAR DIFFERENCE EQUATIONS
}

\author{
by BING LIU and JURANG YAN
}

(Received 9th November 1994)

In this paper we are dealing with oscillatory and asymptotic behaviour of solutions of second order nonlinear difference equations of the form

$$
\Delta\left(r_{n-1} \Delta x_{n-1}\right)+F\left(n, x_{n}\right)=G\left(n, x_{n}, \Delta x_{n}\right), n \in N\left(n_{0}\right) .
$$

Some sufficient conditions for all solutions of (E) to be oscillatory are obtained. Asymptotic behaviour of nonoscillatory solutions of $(\mathrm{E})$ is considered also.

1991 Mathematics subject classification: Primary 39A10.

\section{Introduction}

Recently, there has been a lot of interest in the oscillation and nonoscillation of second order difference equations. See, for example, [1-6] and the references cited therein. In this paper, we consider the second order nonlinear difference equation of the form

$$
\Delta\left(r_{n-1} \Delta x_{n-1}\right)+F\left(n, x_{n}\right)=G\left(n, x_{n}, \Delta x_{n}\right)
$$

where $n \in N\left(n_{0}\right)=\left\{n_{0}, n_{0}+1, n_{0}+2, \ldots\right\}$ ( $n_{0}$ is a fixed non-negative integer) and $\Delta$ is the forward difference operator defined by $\Delta x_{n}=x_{n+1}-x_{n}$. Moreover, $F$ and $G$ are real-valued functions with $x: N\left(n_{0}\right) \rightarrow \mathbf{R}, \quad r: N\left(n_{0}\right) \rightarrow(0,+\infty), \quad F: N\left(n_{0}\right) \times R \rightarrow R \quad$ and $G: N\left(n_{0}\right) \times \mathbf{R}^{2} \rightarrow \mathbf{R}$.

The purpose of this paper is to establish some new results on the oscillatory and asymptotic behaviour of solutions of $(E)$. Our results differ greatly from those in [1-6] and the known literature.

As is customary (see [3], [4] and [6]), a nontrivial solution $\left\{x_{n}\right\}$ of (E) is said to be oscillatory if for every $N>0$ there exists a $k \geqq N$ such that $x_{k} x_{k+1} \leqq 0$. Otherwise the solution is called nonoscillatory.

In this paper, we further assume that the following conditions hold:

$(H)$ There exist sequences $\{f(n)\},\{g(n)\}$ and ratio $m$ of two odd integers such that for all sufficiently large $n$ 


$$
\frac{F(n, u)}{u^{m}} \geqq f(n) \quad \text { for } u \neq 0,
$$

and

$$
\frac{G(n, u, v)}{u^{m}} \leqq g(n) \quad \text { for } u \neq 0
$$

\section{Asymptotic behaviour of nonoscillatory solutions}

In this section, we assume that

$$
\sum_{k=n_{0}}^{\infty}[f(k)-g(k)]=\infty
$$

Theorem 1. Let conditions $(H)$ and $(1)$ hold, then any nonoscillatory solution of $(E)$ must belong to one of the following two types:

$$
\begin{gathered}
A_{c}: x_{n} \rightarrow C \neq 0, n \rightarrow \infty, \\
A_{0}: x_{n} \rightarrow 0, n \rightarrow \infty .
\end{gathered}
$$

Proof. Let $\left\{x_{n}\right\}$ be a nonoscillatory solution of $(E)$, then $x_{n}$ is eventually positive or negative. Thus, from $(E)$, we have

$$
\begin{aligned}
\Delta\left(\frac{r_{n-1} \Delta x_{n-1}}{x_{n-1}^{m}}\right) & =\frac{r_{n} \Delta x_{n}}{x_{n}^{m}}-\frac{r_{n-1} \Delta x_{n-1}}{x_{n-1}^{m}} \\
& =\frac{x_{n-1}^{m} r_{n} \Delta x_{n}-x_{n}^{m} r_{n-1} \Delta x_{n-1}}{x_{n}^{m} x_{n-1}^{m}} \\
& =\frac{\Delta\left(r_{n-1} \Delta x_{n-1}\right)}{x_{n}^{m}}-\frac{\Delta x_{n-1} m \cdot r_{n-1} \Delta x_{n-1}}{\left(x_{n-1} x_{n}\right)^{m}} \\
& \leqq-[f(n)-g(n)]-\frac{\Delta x_{n-1}^{m} \cdot r_{n-1} \Delta x_{n-1}}{\left(x_{n-1} x_{n}\right)^{m}}
\end{aligned}
$$

By the mean value theorem

$$
\Delta x_{n-1}^{m}=m \xi_{n}^{m-1} \Delta x_{n-1}
$$

where $x_{n-1}<\xi_{n}<x_{n}$ or $x_{n}<\xi_{n}<x_{n-1}$. Thus from (2), (3) we have

$$
\Delta\left(\frac{r_{n-1} \Delta x_{n-1}}{x_{n-1}^{m}}\right) \leqq-[f(n)-g(n)]-\frac{m \xi_{n}^{m-1} \cdot r_{n-1}\left(\Delta x_{n-1}\right)^{2}}{\left(x_{n-1} x_{n}\right)^{m}}
$$




$$
\leqq-[f(n)-g(n)]
$$

Summing (4) from $n_{0}+1$ to $n$, we get

$$
\frac{r_{n} \Delta x_{n}}{x_{n}^{m}} \leqq \frac{r_{n_{0}} \Delta x_{n_{0}}}{x_{n_{0}}^{m}}-\sum_{k=n_{0}+1}^{n}[f(k)-g(k)] .
$$

If $x_{n}$ is eventually positive, then there exists $n_{1} \in N\left(n_{0}\right)$ such that $x_{n}>0$ for $n \in N\left(n_{1}\right)$, thus from (5) and (1) we have

$$
\Delta x_{n}<0 \text { for } n \in N\left(n_{1}\right) .
$$

Hence $x_{n}$ is monotone decreasing, and $\lim _{n \rightarrow \infty} x_{n}=C \geqq 0$, where $C$ is a constant.

If $x_{n}$ is eventually negative, then there exists $n_{2} \in N\left(n_{0}\right)$ such that $x_{n}<0$ for $n_{2} \in N\left(n_{0}\right)$, thus from (5) and (1) we have

$$
\Delta x_{n}>0 \text { for } n \in N\left(n_{2}\right) .
$$

Hence $x_{n}$ is monotone increasing, then $\lim _{n \rightarrow \infty} x_{n}$ exists and $\lim _{n \rightarrow \infty} x_{n}=C \leqq 0$.

Thus any nonoscillatory solution of $(E)$ must belong to the following two types: $A_{c}$ or $A_{0}$. The proof of Theorem 1 is complete.

Theorem 2. Let conditions $(H)$ and (1) hold.

(i) If $m=1$, then a necessary condition for equation $(E)$ to have a nonoscillatory solution $\left\{x_{n}\right\}$ which belongs to $A_{c}$ is that

$$
\sum_{k=n_{1}+1}^{k} \frac{1}{r_{k}} \sum_{i=n_{1}+1}^{\infty}[f(i)-g(i)]<\infty
$$

where $n_{1} \in N\left(n_{0}\right)$ is sufficiently large.

(ii) If $0<m<1$, then a necessary condition for equation (E) to have a nonoscillatory solution $\left\{x_{n}\right\}$ which belongs to $A_{0}$ or $A_{c}$ is also (6).

Proof. (i) if $m=1$, let $\left\{x_{n}\right\}$ be a nonoscillatory solution of $(E)$ which belongs to $A_{c}$. If $C>0$, then $x_{n}$ is eventually positive. From the proof of Theorem 1 , we have that $\Delta x_{n}$ is eventually negative and from (1), there exists $n_{1} \in N\left(n_{0}\right)$ such that $x_{n}>0, \Delta x_{n}<0$, and $\sum_{i=n_{1}+1}^{n}[f(i)-g(i)]>0$ for $n \in N\left(n_{1}\right)$. Summing (4) from $n_{1}+1$ to $n$, it follows that

$$
\frac{r_{n} \Delta x_{n}}{x_{n}} \leqq \frac{r_{n_{1}} \Delta x_{n_{1}}}{x_{n_{1}}}-\sum_{i=n_{1}+1}^{n}[f(i)-g(i)] \leqq-\sum_{i=n_{1}+1}^{n}[f(i)-g(i)],
$$

this is,

$$
\frac{\Delta x_{n}}{x_{n}} \leqq-\frac{1}{r_{n}} \sum_{i=n_{1}+1}^{n}[f(i)-g(i)] .
$$


Let $q(t)=x_{n}+(t-n) \Delta x_{n}, n \leqq t \leqq n+1$. Then $q^{\prime}(t)=\Delta x_{n}<0$, and $0<x_{n+1} \leqq q(t) \leqq x_{n}$ for $n<t<n+1$. Hence

$$
\begin{aligned}
\sum_{k=n_{1}+1}^{n} \frac{\Delta x_{k}}{x_{k}} & =\sum_{k=n_{1}+1}^{n} \int_{k}^{k+1} \frac{q^{\prime}(t)}{x_{k}} d t \geqq \sum_{k=n_{1}+1}^{n} \int_{k}^{k+1} \frac{q^{\prime}(t)}{q(t)} d t \\
& =\sum_{k=n_{1}+1}^{n}[\log q(k+1)-\log q(k)] \\
& =\sum_{k=n_{1}+1}^{n}\left[\log x_{k+1}-\log x_{k}\right] \\
& =\log x_{n+1}-\log x_{n_{1}+1} .
\end{aligned}
$$

Thus from (7) and (8), we have

$$
\begin{gathered}
\sum_{k=n_{1}+1}^{n} \frac{1}{r_{k}} \sum_{i=n_{1}+1}^{k}[f(i)-g(i)] \\
\leqq \log x_{n_{1}+1}-\log x_{n+1},
\end{gathered}
$$

from which letting $n \rightarrow \infty$ and noting $\lim _{n \rightarrow \infty} x_{n}=C>0$, we obtain (6).

(ii) If $0<m<1$, let $\left\{x_{n}\right\}$ be a solution of $(E)$ which belongs to $A_{0}$ or $A_{c}$. As shown in the proof of case $m=1$, we can obtain

$$
\frac{\Delta x_{n}}{x_{n}^{m}} \leqq-\frac{1}{r_{n}} \sum_{i=n_{1}+1}^{n}[f(i)-g(i)]
$$

and

$$
\sum_{k=n_{1}+1}^{n} \frac{\Delta x_{k}}{x_{k}^{m}} \leqq(1-m)\left[x_{n_{1}+1}^{1-m}-x_{n+1}^{1-m}\right]
$$

From (9) and (10) we have

$$
\sum_{k=n_{1}+1}^{n} \frac{1}{r_{k}} \sum_{i=n_{1}+1}^{k}[f(i)-g(i)] \leqq(1-m)\left(x_{n_{1}+1}^{1-m}-x_{n+1}^{1-m}\right),
$$

from which letting $n \rightarrow \infty$, and noting $0<m<1$ and $\lim _{n \rightarrow \infty} x_{n}=0$ or $\lim _{n \rightarrow \infty} x_{n}=C>0$, we obtain (6), that is.

$$
\sum_{k=n_{1}+1}^{\infty} \frac{1}{r_{k}} \sum_{i=n_{1}+1}^{k}[f(i)-g(i)]<\infty
$$

If $\left\{x_{n}\right\}$ is eventually negative, similarly we can show that (6) holds. Thus the proof Theorem 2 is complete. 


\section{Oscillation of solutions}

Theorem 3. Let conditions $(H),(1)$ and the following condition hold,

$$
\sum_{k=n_{1}+1}^{\infty} \frac{1}{r_{k}}=\infty
$$

Then all solutions of $(E)$ are oscillatory.

Proof. Suppose on the contrary that there exists a nonoscillatory solution $\left\{x_{n}\right\}$. Without loss of generality, we assume that $x_{n}$ is eventually positive. From the proof of Theorem 1, we have that $\Delta x_{n}$ is eventually negative and from (1), there exists $n_{1} \in N\left(n_{0}\right)$ such that $x_{n}>0, \Delta x_{n}<0$ for $n \in N\left(n_{1}\right)$ and

$$
\sum_{i=n_{1}+1}^{n}[f(i)-g(i)] \geqq 0 \text { for } n \in N\left(n_{1}\right)
$$

Summing $(E)$ from $n_{1}+1$ to $n$, we have

$$
\begin{aligned}
r_{n} \Delta x_{n} & =r_{n_{1}} \Delta x_{n_{1}}-\sum_{i=n_{1}+1}^{n}\left[F\left(k, x_{k}\right)-G\left(k, x_{k}, \Delta x_{k}\right)\right] \\
& \leqq r_{n_{1}} \Delta x_{n_{1}}-\sum_{k=n_{1}+1}^{n} x_{k}^{m}[f(k)-g(k)] \\
& =r_{n_{1}} \Delta x_{n_{1}}-x_{n}^{m} \sum_{k=n_{1}+1}^{n}[f(k)-g(k)]+\sum_{k=n_{1}+1}^{n-1} \Delta x_{k}^{m} \sum_{i=n_{1}+1}^{k}[f(i)-g(i)] \\
& =r_{n_{1}} \Delta x_{n_{1}}-x_{n}^{m} \sum_{k=n_{1}+1}^{n}[f(k)-g(k)]+\sum_{k=n_{1}+1}^{n-1}\left(m \xi_{k}^{m-1} \Delta x_{k}\right) \sum_{i=n_{1}+1}^{k}[f(i)-g(i)]
\end{aligned}
$$

where $x_{k+1}<\xi_{k}<x_{k}$.

From $x_{n}>0, \Delta x_{n}<0$ for $n \in N\left(n_{1}\right)$ and (12), we have

Thus

$$
r_{n} \Delta x_{n} \leqq r_{n_{1}} \Delta x_{n_{1}} .
$$

$$
\Delta x_{n} \leqq \frac{1}{r_{n}} r_{n 1} \Delta x_{n_{1}}
$$

Summing (13) from $n_{1}+1$ to $n-1$, we get

$$
x_{n} \leqq x_{n_{1}+1}+r_{n 1} \Delta x_{n_{1}} \sum_{k=n_{1}+1}^{n-1} \frac{1}{r_{k}}
$$


from (14), letting $n \rightarrow \infty$ and using (11) and $\Delta x_{n_{1}}<0$, we have $x_{n} \rightarrow-\infty$, which contradicts $x_{n}>0$. Thus Theorem 3 is proved.

Theorem 4. Let conditions $(H)$ with $m=1,(11)$ and the following conditions hold,

(i) There exists a sufficiently large $n_{1} \in N\left(n_{0}\right)$ such that for $n \in N\left(n_{1}\right), f(n)-g(n) \geqq 0$ and

$$
\sum_{k=n_{1}+1}^{\infty}[f(k)-g(k)]<\infty
$$

(ii) There exists positive sequence $\left\{C_{n}\right\}$ such that

$$
\sum_{k=n_{1}+1}^{\infty} C_{k}[f(k)-g(k)]=\infty
$$

and

$$
\sum_{k=n_{1}+1}^{\infty} \frac{\left(\Delta C_{k-1}\right)^{2}}{C_{k}\left(\frac{1}{r_{k}-1} \sum_{i=k}^{\infty}[f(i)-g(i)]\right)}<\infty .
$$

Then all solutions of $(E)$ are oscillatory.

Proof. Suppose that there exists a nonoscillatory solution $\left\{x_{n}\right\}$. Without loss of generality, we assume that $x_{n}>0$ for $n \in N\left(n_{1}\right)$. Hence (4) holds. Now, we show that $\Delta x_{n}<0$ for sufficiently large $n$ and that this will lead to a contradiction.

Case (a). If there exists $n_{2} \in N\left(n_{1}\right)$ such that $\Delta x_{n_{2}}=0$, then summing (4) from $n_{2}+1$ to $n$, we have

$$
\begin{aligned}
\frac{r_{n} \Delta x_{n}}{x_{n}} & \leqq \frac{r_{n_{2}} \Delta x_{n_{2}}}{x_{n_{2}}}-\sum_{k=n_{2}+1}^{n}[f(k)-g(k)] \\
& =-\sum_{k=n_{2}+1}^{n}[f(k)-g(k)] .
\end{aligned}
$$

Thus from (15), we have $\Delta x_{n}<0$ for $n \in N\left(n_{2}\right)$. Hence summing $(E)$ from $n_{3} \in N\left(n_{2}\right)$ to $n$, we can obtain that

$$
\lim _{n \rightarrow \infty} x_{n}=-\infty
$$

which contracts $x_{n}>0$.

Case (b) If $\Delta x_{n}>0$ for $n \in N\left(n_{1}\right)$. Similarly to (4) we have 


$$
\Delta\left(\frac{r_{n-1} \Delta x_{n-1}}{x_{n-1}}\right)<-[f(n)-g(n)]
$$

Summing (18) from $n+1, n \in N\left(n_{1}\right)$, to $N$ and letting $N \rightarrow \infty$, we have

Thus

$$
0 \leqq \lim _{N \rightarrow \infty} \frac{r_{N} \Delta x_{N}}{x_{N}} \leqq \frac{r_{n} \Delta x_{n}}{x_{n}}-\sum_{k=n+1}^{\infty}[f(k)-g(k)] .
$$

$$
\sum_{k=n+1}^{\infty}[f(k)-g(k)] \leqq \frac{r_{n} \Delta x_{n}}{x_{n}}
$$

From $\Delta x_{n}>0$ for $n \in N\left(n_{1}\right)$, we have

$$
\frac{1}{r_{n}} \sum_{k=n+1}^{\infty}[f(k)-g(k)] \leqq \frac{1}{x_{n_{1}}} \Delta x_{n} .
$$

Hence

$$
\begin{aligned}
& \Delta\left(\frac{r_{n-1} C_{n-1} \Delta x_{n-1}}{x_{n-1}}\right)=\frac{r_{n} C_{n} \Delta x_{n}}{x_{n}}-\frac{r_{n-1} C_{n-1} \Delta x_{n-1}}{x_{n-1}} \\
& \quad=\frac{C_{n}\left(r_{n} \Delta x_{n}-r_{n-1} \Delta x_{n-1}\right)}{x_{n}}+\frac{C_{n} r_{n-1} \Delta x_{n-1}}{x_{n}}-\frac{r_{n-1} C_{n-1} \Delta x_{n-1}}{x_{n-1}} \\
& \quad=C_{n} \frac{G\left(n, x_{n}, \Delta x_{n}\right)-F\left(n, x_{n}\right)}{x_{n}}-\frac{C_{n} r_{n-1}\left(\Delta x_{n-1}\right)^{2}}{x_{n} x_{n-1}}+\frac{\Delta C_{n-1} r_{n-1} \Delta x_{n-1}}{x_{n-1}} \\
& \leqq-C_{n}[f(n)-g(n)]-\frac{r_{n-1} x_{n}}{x_{n 1}}\left[\frac{\sqrt{C_{n}} \Delta x_{n-1}}{x_{n}}-\frac{\left.\Delta C_{n-1}\right]^{2}}{2 \sqrt{C_{n}}}\right]^{r_{n-1}\left(\Delta C_{n-1}\right)^{2}} \frac{x_{n}}{4 C_{n}} \\
& x_{n-1} \\
& \leqq-C_{n}[f(n)-g(n)]+\frac{r_{n-1}\left(\Delta C_{n-1}\right)^{2}}{4 C_{n}} \cdot \frac{x_{n}}{x_{n-1}} \\
& \leqq-C_{n}[f(n)-g(n)]+\frac{r_{n-1}\left(\Delta C_{n-1}\right)^{2}}{4 C_{n}} \\
& \quad=-C_{n}[f(n)-g(n)]+\frac{r_{n-1} \Delta x_{n-1} \cdot\left(\Delta C_{n-1}\right)^{2}}{4 C_{n} \cdot \Delta x_{n-1}}
\end{aligned}
$$

Summing the following inequality from $n_{1}+1$ to $n+1$,

$$
r_{k-1} \Delta x_{k-1} \leqq-x_{k}[f(k)-g(k)],
$$


we find that

$$
\begin{aligned}
r_{n-1} \Delta x_{n-1} & \leqq r_{n_{1}} \Delta x_{n_{1}}-\sum_{k=n_{1}+1}^{n-1} x_{k}[f(k)-g(k)] \\
& \leqq x_{n_{1}} \Delta x_{n_{1}}=M_{0} .
\end{aligned}
$$

Using (21), (19), and (20) we have

$$
\begin{aligned}
& \Delta\left(\frac{r_{n-1} C_{n-1} \Delta x_{n-1}}{x_{n-1}}\right) \\
& \leqq-C_{n}[f(n)-g(n)]+\frac{M_{0} \cdot\left(\Delta C_{n-1}\right)^{2}}{4 x_{n 1} \cdot C_{n}\left(\frac{1}{r_{n-1}} \sum_{k=n}^{\infty}[f(k)-g(k)]\right)} \\
& =-C_{n}[f(n)-g(n)]+M \cdot \frac{\left(\Delta C_{n-1}\right)^{2}}{C_{n}\left(\frac{1}{r_{n-1}} \sum_{k=n}^{\infty}[f(k)-g(k)]\right)},
\end{aligned}
$$

where $M=M_{0} / 4 x_{n_{1}}$. Summing (22) from $n_{1}+1$ to $n$, we have

$$
\begin{gathered}
\frac{r_{n} C_{n} \Delta x_{n}}{x_{n}} \leqq \frac{r_{n_{1}} C_{n_{1}} \Delta x_{n_{1}}}{x_{n_{1}}}-\sum_{k=n_{1}+1}^{n} C_{k}[f(k)-g(k)] \\
+M \sum_{k=n_{1}+1}^{n} \frac{\left(\Delta C_{n-1}\right)^{2}}{C_{k}\left(\frac{1}{r_{k-1}} \sum_{i=k}^{\infty}[f(i)-g(i)]\right)} .
\end{gathered}
$$

Letting $n \rightarrow \infty$ and noting (16), (17), we get

$$
\lim _{n \rightarrow \infty} \frac{r_{n} C_{n} \Delta x_{n}}{x_{n}}=-\infty .
$$

Thus there exists $n_{2} \in N\left(n_{1}\right)$ such that $\Delta x_{n}<0$ for $n \in N\left(n_{2}\right)$, which contradicts $\Delta x_{n}>0$ for $n \in N\left(n_{1}\right)$.

Thus from Cases (a) and (b) we can show that there exists $n_{3} \in N\left(n_{1}\right)$ such that $\Delta x_{n_{3}}<0$. Summing (4) from $n_{3}+1$ to $n$ we have

$$
\frac{r_{n} \Delta x_{n}}{x_{n}} \leqq \frac{r_{n_{3}} \Delta x_{n_{3}}}{x_{n_{3}}}-\sum_{k=n_{3}+1}^{n}[f(k)-g(k)] \text {. }
$$

Hence $\Delta x_{n}<0$ for $n \in N\left(n_{3}\right)$. Similarly to the last part of the proof of Theorem 3 and from (11) we have $\lim _{n \rightarrow \infty} x_{n}=-\infty$, which contradicts $x_{n}>0$. Theorem 4 is proved. 
For the purpose of illustration we consider the following example.

Example. Consider the difference equation

$$
\Delta\left(\frac{1}{2 n^{1+\delta}} \Delta x_{n-1}\right)+\frac{1}{n^{1+\delta}} x_{n}+\frac{1}{4(n+1)^{1+\delta}}\left(\Delta x_{n}\right)^{2}=0, n \in N\left(n_{0}\right), n_{0} \geqq 1
$$

where $0<\delta<1$. Let $C_{n}=n, f(n)=1 / n^{1+\delta}$ and $g(n)=0, n \in N\left(n_{0}\right)$, then we find that conditions $(H),(11)$, and (15) $-(17)$ are satisfied. Thus from Theorem 4 all solutions of $(E)$ are oscillatory. In fact, $\left\{x_{n}\right\}=\left\{(-1)^{n}\right\}$ is such a solution. We believe that the conclusion is not deducible from the oscillation criteria in $[3,4,6]$ and the known literature.

Acknowledgements. The authors thank the referee for many valuable suggestions.

\section{REFERENCES}

1. Sui-Sun Cheng and Horng-JaAn Li, Bounded and zero convergent solutions of second order difference equations. J. Math. Anal. Appl. 141 (1989), 463-483.

2. Andrzes Drozdowicz and Jerzy Popenda, Asymptotic behavior of solutions of difference equation of second order, J. Comput. Appl. Math. 47 (1993), 141-149.

3. Hue-Zhong $\mathrm{He}$, Oscillatory and asymptotic behavior of second order nonlinear difference equations. J. Math. Anal. Appl. 175 (1993), 482-498.

4. Zdzislaw Szafranski and Blazes Szmanda, Oscillatory behavior of difference equations of second order, J. Math. Anal. Appl. 150 (1990), 414-424.

5. William F. TrenCh, Asymptotic behavior of solutions of Emden-fowler difference equations with oscillating coefficients, J. Math. Anal. Appl. 179 (1993), 135-153.

6. B. G. ZHANG, Oscillation and asymptotic behavior of second order difference equations, $J$. Math. Anal. Appl. 173 (1993), 58-68.

DePaRTMENT OF MATHEMATICS

HUBEI NORMAL UNIVERSITY

HUANGSHI, HUBEI 435002

People's Republic of China
Department of Mathematics

SHANXI UNIVERSITY

Taiyuan, ShanXi 030006

People's Republic of China 